\title{
Leprosy elimination - sprint or marathon?
}

\author{
RICHARD CROFT \\ 70 Culver Lane, Reading, Berks, RG6 1DY
}

\section{Accepted for publication 1 October 1999}

The widespread implementation of leprosy elimination campaigns (LECs) as a tool to reduce the pool of undetected leprosy cases in highly endemic countries is a very welcome and important development. Not only is the exercise proving successful in terms of new case detection, but it has brought the cause of leprosy elimination into sharp focus in those countries right from high government level down to grassroots health delivery systems and the general public. Leprosy is on the agenda in cultures where it has been difficult even to name. However, in the midst of all this it is worth reflecting on some of the weaknesses of the programme. Broadly, these relate to organizational issues, training quality and disability.

LECS have been implemented rather monolithically, with blanket coverage of all areas in the target country. However, there may be wide disparity in the quality of leprosy control services across the country. The campaigns will probably be most efficiently managed in those areas where leprosy control services are best developed, but arguably, these are also the areas that need them least. Conversely, areas with poorly organized leprosy control services are the areas least likely to run the campaigns well, but they may also be the areas where there is the greatest potential impact. It would be good to introduce more flexibility, enabling limited resources to be more focussed in areas where leprosy services have been poor.

Reaching the goal of eliminating leprosy from endemic countries will depend more on the long-term commitment of grassroots level health workers than short, sharp bursts of high-level publicity, although an LEC can inject a much needed shot in the arm for dispirited staff. It is easier to run a sprint than a marathon, but that should not blind us to the need to seek to continue to resource, train and motivate doctors and paramedical staff who are responsible for the day-to-day management of leprosy control services, whether in an integrated, horizontal programme or a dedicated vertical one. Frequently, when the glare of publicity has passed, the detection and treatment of leprosy patients falls to the bottom of the list of priorities. It is often the case that countries with a significant leprosy burden are also those struggling with other serious (and sometimes more immediate) health problems - tuberculosis, AIDS, malaria and overpopulation, for example.

The overall effectiveness of an LEC depends critically on the quality of training given to those who will administer it. In some cases, the emphasis in training may be simply on 'getting it over with', and with limited time (training courses' length varies from 1 day for

Correspondence to: R. Croft (e-mail: richard@crofts32.freeserve.co.uk) 
health workers to 6 days for medical officers) and resources available, the outcome can be variable. Impressive-sounding statistics ('forty thousand health workers have been trained') simply relate to the number of bodies who have sat through training sessions. Audit of training quality has been very limited.

The bottom line for training is the correct diagnosis of leprosy and the correct administration of MDT. The question of overdiagnosis of leprosy cases is a thorny one. In many LEC situations skin smears are not routinely used to aid diagnosis, and supervision is very limited. In addition, there is pressure to come up with results. In such a situation, it would be surprising if overdiagnosis did not take place. Further, the addition of single-dose ROM/MDT to the therapeutic armamentarium is likely to compound the problem of overdiagnosis since it is so easy to give, and side-effects are negligible. Why not give it and be on the safe side? Since the question of overdiagnosis has not (to my knowledge) been satisfactorily assessed, it may be premature to make too much of the large numbers of new cases being reported.

Related to both organization and training is the matter of MDT completion. Since case detection and MDT administration does not necessarily equal MDT completion (except for single-dose ROM/MDT), it would be instructive to ascertain completion rates for cases detected and treated during an LEC. It is at this point that the 'rubber hits the road', since completion will depend very much on the internal motivation of health workers to finish the job, adequate and informed supervision, available resources, and the critical outcome of the contest between leprosy and other urgent and important health problems on the agenda.

LECs have been very focussed on case detection and treatment, with much justification, of course. There has, however, been much less emphasis on the management of nerve damage and secondary disability, although it is patients who experience these complications who will have really suffered the consequences of leprosy. On the whole, management of these problems remains the province of specialist programmes which tend to be more sparsely distributed.

Widespread LEC implementation, combined with shorter courses of MDT, will certainly bring the point prevalence of leprosy to below or near to the elimination goal of 1 case per 10,000 population by the dawn of the third millennium in most endemic countries. But what then? It is so important that leprosy control and the management of disability be continued well into the next century, and that knowledge and enthusiasm for leprosy work is maintained. One hundred-metre sprinters can finish the short course in a few seconds, but the real race is a marathon, which takes much longer to run. 